\title{
Filigrane
}

Écoutes psychothérapiques

\section{Moi, toi et... ou est passé le roi ? Les voies de la socialisation précoce}

\section{Irène Krymko-Bleton}

Volume 16, numéro 2, automne 2007

Les hauts lieux et non-lieux du rêve II

URI : https://id.erudit.org/iderudit/016923ar

DOI : https://doi.org/10.7202/016923ar

Aller au sommaire du numéro

Éditeur(s)

Revue Santé mentale au Québec

ISSN

1192-1412 (imprimé)

1911-4656 (numérique)

Découvrir la revue

Citer cet article

Krymko-Bleton, I. (2007). Moi, toi et... ou est passé le roi ? Les voies de la socialisation précoce. Filigrane, 16(2), 76-96. https://doi.org/10.7202/016923ar
Résumé de l'article

L'article présente les aspects contextuels de pratique clinique avec des enfants dégagés à partir de la pratique préventive d'accueil des bébés et leurs familles dans le cadre informel de La Maison buissonnière. Cette pratique place le bébé en interlocuteur principal. La famille, voire la société, sont alors interrogées autant que possible, depuis sa place à lui. Les débats qui concernent les valeurs sociétales, la délinquance, l'éducation et la psychopathologie trouvent alors leur point de convergence dans le concept de socialisation précoce que l'équipe de La Maison buissonnière a mis au centre de sa réflexion. 


\title{
Moi, toi et... ou est passé le roi? Les voies de la socialisation précoce
}

\author{
irène krymko-bleton
}

\begin{abstract}
L'article présente les aspects contextuels de pratique clinique avec des enfants dégagés à partir de la pratique préventive d'accueil des bébés et leurs familles dans le cadre informel de La Maison buissonnière. Cette pratique place le bébé en interlocuteur principal. La famille, voire la société, sont alors interrogées autant que possible, depuis sa place à lui. Les débats qui concernent les valeurs sociétales, la délinquance, l'éducation et la psychopathologie trouvent alors leur point de convergence dans le concept de socialisation précoce que l'équipe de La Maison buissonnière a mis au centre de sa réflexion.
\end{abstract}

$F$ iligrane m'invite à présenter à son lectorat mon expérience de travail clinique auprès de bébés et leurs familles pour le sensibiliser aux certains enjeux de la socialisation du petit enfant. Cette invitation m'honore mais m'oblige à reparcourir mon trajet de clinicienne, qui a débuté par le travail avec des délinquants enfermés dans une institution carcérale et m'a conduit par la suite, via la pratique avec des enfants psychotiques dans un grand hôpital montréalais, vers ce qui semble être son aboutissement - la pratique clinique avec des bébés et leurs familles dans le cadre informel de la Maison buissonnière ${ }^{1}$. Celle-ci, lieu de socialisation précoce et de prévention primaire, fête cette année ses 15 ans d'existence officielle.

Mon travail de recherche universitaire et ma pratique privée auprès des adultes et des familles s'articulent étroitement avec ces trois domaines d'expérience qui ont balisé mon chemin et qui ont pour point commun de convoquer le personnage du père sur le devant de la scène. Ils ont aussi en commun de situer la pratique clinique en une étroite dépendance du contexte social et institutionnel dans lequel elle se déroule.

\section{Pour la petite histoire}

Arrivée au Québec au début des années soixante-dix, jeune psychologue, j'ai été frappée par ce que j'ai appelé ultérieurement «la complainte du père absent» (1990). Le discours social concernant la famille me semblait organisé autour de cette image du père déprécié, absent de parole. Engagée dans le travail clinique avec des adolescents délinquants puis avec des enfants psychotiques, je devais constater que très souvent son absence était aussi physique. Ce qui me frappait néanmoins encore d'avantage était le glissement progressif de ce discours sociétal 
sur l'absence du père et la complainte la concernant vers l'affirmation de l'inutilité de ce père - affirmation à laquelle le concept lacanien du Nom du Père opposait, dans les cercles des intervenants cliniques, une réfutation évidemment inopérante à cause de ses relents catholico-patriarcaux.

Lors des années qui ont suivi, j'ai entrepris à l'université les recherches articulées autour de questions qui concernaient la place du père dans la famille et la société québécoises (1985, 1989, 1990). Je souhaitais aussi créer un programme de soutien pour les hommes qui, selon mon idée de l'époque, n'arrivaient pas à s'autoriser à être des pères de leurs enfants. Je retrouve aujourd'hui les échos de mes étonnements de ces années-là dans des articles récents écrits par les chercheurs et intervenants qui ont continué de s'occuper des pères et des hommes québécois (notamment Lacharité 2004, Dulac 2000, Gagnon 2006). J'y reviendrai.

Quant à moi, le hasard des rencontres a décidé autrement de l'orientation future de mes efforts. Marie-Françoise Liaume-El Khouri qui arrivait au Québec, forte de son expérience de travail à La Maison Verte ${ }^{2}$ mise sur pied à Paris par Françoise Dolto et ses collaborateurs, a su me convaincre - au bout de deux ans d'un séminaire privé que j'avais organisé autour de la problématique paternelle - que travailler avec des nourrissons et des enfants très jeunes, dans ce moment de déséquilibre de la dynamique familiale que la naissance d'un enfant provoque, était d'une plus grande pertinence. De cette rencontre, et suite à ce séminaire, est né le projet de socialisation précoce pour les enfants d'âge pré-œdipien et, subséquemment, l'organisme à but non lucratif La Maison buissonnière. Vingt ans plus tard, il s'y poursuit un travail clinique et théorique autour des questions de la structuration de soi chez l'enfant et de ses rapports avec le discours et les pratiques sociales actuelles $^{3}$. La question paternelle, ou si l'on préfère, la question de la nature du processus de séparation entre l'enfant et la mère, s'y retrouve de façon insistante.

Or, au moment où le concept d'attachement occupe tout l'espace de recherche et d'intervention auprès de l'enfant très jeune, mettre l'accent sur la socialisation, donc sur la séparation, rencontre peu d'intérêt, même si la mise en place du réseau de garderies à prix réduit devrait en principe orienter l'intérêt des intervenants et des chercheurs vers les questions de la séparation ${ }^{4}$.

La psychanalyse a insisté principalement sur la triangulation fantasmatique de relations mère-enfant, qui précède la naissance de l'enfant et l'introduit après celle-ci à son devenir social. Le concept du Nom du père a été convoqué pour préciser les conditions nécessaires pour qu'un enfant ne devienne pas psychotique. Mais tous les déboires de socialisation et tous les dérapages de la fonction paternelle ne rendent pas nécessairement des enfants psychotiques. Les représentations complexes de ce qu'est un homme ou une femme, de ce qu'est un enfant, de ce qu'une société permet ou défend, l'histoire familiale, les accidents de la vie, les idéologies en cours dans une société donnée, les institutions sociales disponibles orientent les modalités de la socialisation de l'enfant, ordonnent ses ratées ou ses réussites. Si les idéologies offrent les matériaux qui cimentent des fantasmes, les institutions offrent le cadre à leur mise en scène. 


\section{La Maison buissonnière}

Les références à la triangulation œdipienne dans ses effets transgénérationnels, la théorisation par Françoise Dolto du développement psychique de l'enfant formulée dans sa théorie de l'image inconsciente du corps et des castrations symboligènes ainsi que la conceptualisation par Winnicott des débuts de la pensée et des processus créateurs (de l'expérience culturelle) sont les principaux repères pour la pratique clinique à la Maison buissonnière.

Cette pratique a des effets préventifs. Dans le contexte actuel, alors que le mot «prévention» équivaut aux seules pratiques de dépistage et d'intervention éducative, il est nécessaire de préciser que notre conception de la prévention s'appuie sur les principes opposés à l'idéologie interventionniste qui préside à ces procédés.

Lieu de rencontres, la Maison buissonnière laisse ses portes ouvertes à qui veut bien venir et respecte l'anonymat de ses visiteurs. On y trouve absurde de vouloir remédier aux difficultés entre la mère et son enfant en apprenant à la mère des gestes et des comportements adéquats, ce vers quoi ont mené les applications récentes de la théorie de l'attachement dans le réseau de santé au Québec. C'est un truisme de dire que la naissance de son enfant renvoie la mère à sa propre préhistoire, autant dans le sens généalogique (la place qui est la sienne dans sa famille d'origine) que dans le sens relationnel (comment elle était portée par sa propre mère et soignée lorsqu'elle était bébé); un autre que sa capacité de prendre soin de son enfant en dépend. On oublie toutefois souvent de rajouter que cette capacité est aussi déterminée par la façon dont cette mère est entendue et accueillie (le père de l'enfant également) à ce moment-charnière par des interlocuteurs possibles et quel support affectif et effectif est offert au couple de parents.

Le moyen de prévention que la Maison buissonnière préconise est une écoute attentive, mais pas insistante, offerte aux enfants et leurs accompagnateurs adultes par une multitude d'interlocuteurs potentiels que constituent les équipes $\mathrm{d}^{\prime}$ «accueillants». Le travail de l'appropriation théorique et du développement conceptuel effectué par l'ensemble du groupe des «accueillants» dans les coulisses de la pratique garantit l'amélioration progressive de conditions de cette écoute. L'organisation des équipes d'accueil par trois intervenants vise l'évitement de collusions duelles à tous les niveaux. La présence d'au moins un homme par équipe rompt avec l'univers exclusivement féminin et maternel. L'expérience acquise avec le temps permet de reprendre l'objectif de «parler vrai » aux enfants, cher à Dolto, sans provoquer des effets de violence qu'induisent dans le champs social ces interprétations sauvages que nombre d'interlocuteurs sociaux produisent sans s'en rendre compte.

Le débat sur l'évolution de la société d'aujourd'hui est d'une très grande complexité et paraît souvent confus, tellement sont grandes et rapides ses transformations. La pratique avec des nourrissons et des enfants tout petits au moment où leurs parents essayent de retrouver un équilibre perturbé par leur naissance, éclaire d'une lumière crue bien des enjeux sociétaux. Dans ce texte, je reprendrai comme 
trame de réflexion les fils de mes intérêts passés (la place du père et la délinquance) pour situer des enjeux de la prévention par la socialisation précoce.

\section{La question du Père: bref retour sur l'œdipe}

Une maman fréquente avec grande assiduité un de nos points d'accueil avec ses deux enfants. C'est une famille charmante, aimante, ils constituent un trio parfait. Nous ne voyons jamais le papa qui, selon le mot de son fils, «est à l'école», mais connaissons son existence puisque la maman le mentionne souvent. Les enfants, lorsqu'ils ont appris à parler sont devenus automatiquement bilingues - la maman parle avec eux en français, mais à la maison tout le monde parle une autre langue, qui est la langue d'origine du père. Un jour, à l'occasion d'un jeux, l'un des nous ${ }^{5} \mathrm{~s}$ 'aperçoit que l'aîné qui a environ trois ans et sait déjà compter jusqu'au 10, saute systématiquement le chiffre 4 . Il compte donc : 1, 2, 3, 5, 6... La maman confirme en riant qu'effectivement c'est toujours comme cela quand il compte en français, alors que dans l'autre langue tous les chiffres y sont. Après réflexion, nous expliquons au petit garçon que même si son papa n'est pas là en français, il est dans le cœur de sa maman tout le temps. De toute façon, il ne peut pas le remplacer, même si le papa est à l'école, puisqu'il est son papa (et pas un frère aîné, par exemple) et que lui-même est son fils. Nous invitons la maman à raconter à l'enfant l'histoire de sa rencontre avec son mari et celle de sa naissance à lui. Elle se propose de le faire au moment du coucher puisque l'enfant adore l'histoire de la rencontre et du mariage de son oncle préféré. Nous apprenons néanmoins qu'il n'a pas vraiment apprécié le changement du récit et qu'il est devenu capricieux et irrité contre la maman. Après une ou deux semaines, les choses sont rentrées en ordre, le chiffre quatre faisant son apparition en français d'abord timidement et ensuite de façon fluide.

\section{Enfance et société}

En contraste avec cette petite histoire, troubles d'apprentissage divers, hyperactivité, troubles d'attention... : nombre de problèmes dont l'enfance de ce début de $\mathrm{XXI}^{\mathrm{e}}$ siècle semble accablée dans des pays occidentaux, diagnostiqués sans discernement, est obstinément traité par des médications de plus en plus précoces ou par des techniques de réhabilitation qui ne tiennent aucun compte des déboires affectifs des enfants. Il n'est pas plus facile d'être enfant aujourd'hui que cela ne l'était hier. Pourtant nous savons aujourd'hui que bien de problèmes trouvent leur 
origine dans de petits malentendus de l'enfance ou s'enracinent de façon plus pernicieuse dans des séparations mal préparées et mal vécues.

La disposition de l'enfant de rester dans une relation exclusive et fusionnelle avec sa mère est conflictuelle avec sa tendance à vouloir devenir grand et autonome - à l'image de ceux qui s'occupent de lui, mais aussi à l'image des enfants un peu plus grands et plus habiles que lui, qui l'attirent irrésistiblement. De son côté, après la séparation de la naissance, la mère entreprend un travail de distanciation et de différentiation psychiques avec lui. C'est là où le père, ou plus exactement l'homme de la mère, instance tierce, donc séparatrice et socialisante dans sa relation à l'enfant, peut lui faire cruellement défaut.

De façon générale, la société actuelle offre de moins en moins des choix relationnels aux enfants pour leur permettre de s'extraire de la relation initiale à la mère. La contestation par la société de structures familiales traditionnelles les expose, encore d'avantage aujourd'hui que dans le passé, à la toute-puissance (en tout cas supposée par eux) maternelle. Les mères, elles-mêmes grandies dans une société «sans pères », sont également de plus en plus perdues.

Les problèmes, petits ou grands, que nous rencontrons tous les jours à La Maison buissonnière et qui constituent des achoppements au futur fonctionnement social des enfants nous éclairent sur le lien entre les idéologies dominantes ayant cours dans notre société et les soins donnés aux bébés. Elles nous éclairent en premier lieu, en deçà des discours, sur les impasses de la sexualité des adultes et de leurs liens avec les pratiques domestiques qui affectent des enfants.

\section{La question sexuelle à la Maison buissonnière}

À la Maison buissonnière, la question sexuelle se profile le plus souvent de façon la plus banale à propos de pratiques très courantes comme l'usage du lit parental ou l'usage des toilettes.

\section{Le lit ou la sexualité empêchée}

Pratique très courante que celle consistant à laisser dormir les enfants dans le lit parental pendant des mois, voire des années après leur venue au monde. Tous ceux qui travaillent avec des enfants savent qu'il n'est pas rare que les enfants restent dans le lit de leur mère (ou de leurs parents) jusqu'à l'âge de 6, 7 ans. Pour certaines mères, infliger une séparation pour la nuit à l'enfant, alors que les adultes dorment ensemble, semble incompréhensible. Elles évoquent souvent les habitudes africaines, oubliant simplement que chaque société africaine réglemente étroitement ses pratiques domestiques ${ }^{6}$. D'autres, lorsque le conjoint n'est pas là, ont peur la nuit et se sentent mieux avec le petit corps de l'enfant près d'elles. D'autres encore se plaignent de la situation, mais n'arrivent pas à la changer : c'est l'enfant qui décide. La configuration que j'ai trouvée la plus cocasse à La Maison buissonnière était celle d'un garçon de 18 mois qui dormait toujours avec sa mère. Celle-ci disait ne pas aimer s'endormir seule dans son lit. Le père, qui travaillait tard, dormait sur le divan du salon (c'est la place réservée le plus souvent aux pères 
dans ce genre de situation). Et c'est le chat de la famille qui dormait dans le lit acheté à grands frais lors des préparatifs pour l'arrivée de l'enfant !

Il ne faut pas croire, que ce sont les mères seules qui orchestrent un tel arrangement rendant difficile voire impossible la vie sexuelle. Les hommes perdent souvent tout désir sexuel pour leur compagne devenue mère et s'accommodent fort bien du canapé. La transformation de leur partenaire sexuelle en mère la frappe de l'interdit de l'inceste. Ils ne désirent donc nullement retrouver leur place dans le lit conjugal.

L'enfant peut néanmoins dormir aussi avec ses deux parents. J'ai déjà rencontré une jeune fille de 13 ans qui dormait encore avec son papa et sa maman. La psychologue scolaire qui la rencontrait pour des problèmes d'apprentissage et des difficultés relationnelles n'avait rien à redire sur cette situation puisque la jeune fille affirmait qu'elle avait peur de dormir seule.

On peut lire dans Le Grand Robert qu'abuser veut dire outrepasser ses droits ou ses pouvoirs et qu'on peut abuser de la complaisance de quelqu'un pour l'assujettir. Cela me semble être le cas en ce qui concerne l'enfant pris en otage pour servir d'excuse à l'impasse sexuelle des parents.

Il est d'ailleurs évident que sauf exception, une telle impasse ne peut durer qu'un temps avant que l'une ou l'autre des parties ne réagisse. Les données statistiques nous éclairent quant à l'issue de ce tremblement de terre qu'est la naissance de l'enfant pour des nombreux couples: les séparations de plus en plus nombreuses dans l'ensemble de la population surviennent de façon la plus fréquente pendant la période de deux premières années de vie de l'enfant ${ }^{7}$. Lors de ces séparations, les problèmes sexuels sont très rarement travaillés, voire abordés et les questions des enfants ne sont pas entendues.

\section{Intimité ou promiscuité: le détour par les toilettes}

L'usage des toilettes révèle à La Maison buissonnière un autre point d'achoppement où des peines de séparation se chevillent à l'érotisme. Les mères (ou parfois les gardiennes) hésitent de laisser l'enfant «seul» dans le local de jeux et tentent de l'amener à la toilette avec elles, parfois sous prétexte d'avoir à lui changer la couche, parce qu'elles savent que nous n'approuvons pas une telle pratique. Nous n'avons jamais vu un père faire la même chose. Peut-être que la crainte d'être accusé d'abus sexuel introduit ici un frein ? Certains enfants réagissent effectivement de façon dramatique à la disparition de leur mère derrière la porte fermée. Il nous arrive d'avoir à déployer des trésors d'argumentation patiente, étalés sur plusieurs semaines, pour que la mère et l'enfant acceptent sans drame ce court moment de séparation! Il nous arrive de nous trouver devant un mur d'incompréhension et de comportements innocemment perverses, que nous ne réussissons pas à traverser.

Une petite fille a fréquenté pendant longtemps La Maison buissonnière. Après une première visite avec ses parents, nous 
ne les avons malheureusement jamais revus: elle venait accompagnée d'une gardienne. Enfant jolie et sage comme une image, toujours habillée de façon recherchée, elle attirait pourtant mon regard à cause d'un manque de fluidité dans ses mouvements - elle habitait son corps avec inconfort. La gardienne, qui l'amenait avec une régularité d'horloge, nous barrait toujours l'accès à l'enfant en l'occupant de façon continue. L'enfant a rapidement compris qu'il lui fallait s'occuper de la gardienne et qu'elle n'avait pas droit de l'abandonner dans son coin. Lorsque la petite a atteint environ trois ans, elle a tenté sans succès de jouer au docteur avec la gardienne et toucher sa poitrine avec le stéthoscope de la trousse de jeux. J'ai essayé, aussi sans succès, de savoir si elle rejouait ainsi une visite médicale récente ou si elle posait une question sur la féminité. La gardienne a évité les gestes de l'enfant avec gêne et le jeu s'est arrêté après quelques tentatives infructueuses. Et pourtant, cette femme prude à tous les égards l'amenait régulièrement avec elle aux toilettes. Elle y filait lorsque nous avions les yeux tournés et mentait en répondant à nos questions. Nous n'avons pas réussi de savoir si elle faisait la même chose à la maison. Fort courtoise et souriante, elle était absolument imperméable à nous. Finalement, après l'apparition d'un petit cousin dans sa vie, la petite fille a trouvé une stratégie d'opposition - elle n'a plus joué qu'à des jeux de garçons. Nous lui avons parlé de différence de sexes et de devenir des petites filles et des petits garçons en sachant que cette fois, elle nous écoutait, mais sans réaction apparente. Nous espérions qu'à la longue, sa stratégie ne s'avérerait pas trop coûteuse pour elle.

\section{D'où viennent les enfants? Embêtante histoire de la petite graine}

D'une façon plus évidente pour tous, la question sexuelle se manifeste à La Maison buissonnière par la présence de mamans enceintes. Les enfants soulèvent les questions qui se rapportent à la grossesse directement ou, le plus souvent, par leurs jeux. Par exemple, la présence d'une maman enceinte peut provoquer une cascade de jeux sur le thème «comment est-il rentré là ?» et «comment va-t-il sortir?». Pour peu que l'on s'en aperçoive et qu'on commente la question, des résistances sourdes se font sentir chez beaucoup de parents. Mais d'une façon qui nous semble toujours un peu magique, une fois la question ouverte, elle se répercute d'un enfant à l'autre, d'un jeu à l'autre. Elle s'élabore et se symbolise lorsque les parents, finalement amusés, l'autorisent. Il arrive néanmoins des situations qui nous rappellent à quel point la sexualité s'épanouit plus sur les écrans de télévision que dans la vraie vie. 
Une maman qui nous paraissait assez «libérée» (ses grands décolletés, ses vêtements sexy nous avaient induits en erreur), a révélé un jour sa gêne et son incapacité d'annoncer à son fils de 18 mois qu'elle était enceinte de 6 mois déjà. Tout le monde en parlait autour de l'enfant, mais il était censé «ne rien en savoir». Il s'est mis à mordre sa mère, qui a refusé pendant plusieurs semaines de voir un lien entre l'apparition de ce comportement et sa grossesse tue. Lorsque finalement, sous notre influence, elle lui a glissé l'information en s'aidant d'un livre et que je me suis donc sentie autorisée d'en parler avec lui, il m'a mis sa main sur la bouche! Le sujet était tabou et il le savait.

L'histoire la plus populaire est néanmoins celle de la petite graine que des papas mettent dans les ventres des mamans (comme des mamans mettent de la nourriture dans la bouche des enfants?) qui pousse là. Les mamans font pousser des bébés comme la terre fait pousser les plantes; les papas sont des jardiniers.

\section{Hommes, pères et garçons dans la societè quèbècoise}

Denise Bombardier racontait récemment à la télévision avec beaucoup de l'humour comment sa grand-mère traitait ses oncles lorsqu'ils revenaient éméchés de la taverne le samedi soir. Une pichenotte derrière la tête les envoyait en bas de l'escalier pour qu'ils dessoûlent. Matriarcat québécois. Si dans mon article sur la paternité écrit en 1990 je m'appuyais sur une littérature anthropologique et sociologique pour comprendre les aléas de la paternité que j'observais par la lorgnette de la clinique, je convoquerai aujourd'hui quelques écrits de chercheurs en psychologie et d'intervenants qui travaillent avec des groupes d'hommes, puisqu'il est impossible de séparer le sujet de la paternité au Québec de la question plus générale de la place que les hommes occupent dans l'imaginaire féminin et sociétal.

Comme le soulignait Sophie Martinat dans sa thèse doctorale et dans ses écrits (1985, 1987, 1990), l'imaginaire sociétal québécois a été empreint par les aléas d'une histoire marquée par la conquête et par les prérogatives qui ont été par la suite dévolues à l'Église catholique. Selon sa thèse, la religion a magnifié les pouvoirs procréateurs de l'homme tout en le reléguant à la périphérie de l'affectivité familiale. Si à l'époque le père était à la fois omniprésent et inexistant, après la Révolution tranquille on en est arrivé à questionner l'utilité de son existence. À mon sens, l'image de la masculinité de l'homme québécois se fige aussi dans une autre image stéréotypé qui tente de le réhabiliter: celle de coureur de bois. Image qui court de Maria Chapdelaine (1921) de Louis Hémon aux Mâles (1971) de Gilles Carle, image ambivalente: séduisant, certes, le mâle est aussi dangereux et «sauvage». Si le mâle continue donc de provoquer la méfiance, on pourrait peutêtre dire qu'aujourd'hui la médecine (via des inséminations artificielles) et les experts de toutes les sortes: psychiatres, éducateurs, psychologues, diverses agences sociales ont remplacé à la fois le père et le curé. La jonction des discours 
néo-libéral (qui appelle donc à l'obligation de l'épanouissement individuel) et de l'héritage post-féministe (qui condamne toutes les formes de jeux de séduction, jugée dégradante) ne rend pas les choses plus simples.

Germain Dulac ${ }^{8}$ (2000) dénonce la fragilité de la paternité dans la société québécoise et évoque le paradoxe de l'appel au père nécessaire (pour l'épanouissement de l'enfant) doublé de l'image du mâle immoral et du parent abject. S'appuyant sur une bibliographie consistante, il rappelle que durant les années soixante-dix on s'interrogeait sur la capacité parentale des pères; on ne lui reconnaissait alors de qualités parentales qu'au prix de la quasi-interchangeabilité des rôles parentaux en insistant que «les papas font aussi de bonnes mamans » comme dans cette citation qu'il rapporte. Mon expérience confirme Dulac: nous n'avons pas encore dépassé ce débat et c'est au prix de renoncement à leur différence sexuelle que les mâles (ou les hommes), qui sont soupçonnés d'être violents par nature, peuvent éventuellement retrouver leurs prérogatives parentales. Carl Lacharité (2004) plaide dans Psychologie Québec pour que la contribution spécifique des pères soit reconnue par la société en général et l'État en particulier. De son côté, Jean-Pierre Gagnon (2006), directeur de recherche de l'organisme L'Après-rupture: Ateliers pour les liens pères-enfants, écrit dans le cadre d'une consultation menée par Le Conseil de la famille et de l'enfance, que négliger le rôle important des pères, c'est «aussi favoriser le mépris de la masculinité et de la paternité trop souvent associés à la violence et à l'irresponsabilité ». L'analyse des statistiques (taux de suicides selon les âges et les sexes, taux de réussites scolaires, etc.) confirme l'impression qu'il n'est pas facile d'être un garçon au Québec. La place me manquant pour engager ici une discussion sur ce sujet, je propose seulement une petite vignette clinique tirée du travail au quotidien avec des tout petits.

Une maman fréquente régulièrement La Maison buissonnière avec un petit garçon et une petite fille. Les deux enfants sont charmants. La maman est très fière de sa petite, «qui saura se défendre dans la vie». La petite fille est en effet aussi charmante qu'affirmative. À un an, quand elle n'obtient pas ce qu'elle veut, elle se plie en deux et elle hurle. Plus tard, elle affronte les autres enfants par un «non! » tonitruant et ne leur cède pas facilement les jouets qui l'intéressent. Plus tard encore elle se met à surveiller et à défendre les jouets avec lesquels joue son frère. Le petit garçon, lorsque quelque chose ne va pas pour lui ne se défend pas, mais devient triste. La maman intervient dès qu'il touche un jouet jugé agressif (par exemple, une épée en plastic), esquisse un geste d'affirmation, etc. Lorsque je lui fais remarquer que sa petite fille est pourtant bien plus agressive que son fils, elle me répond: «Oui, mais la violence... je ne peux vraiment pas supporter». Parce qu'elle a appris à nous faire 
confiance, qu'elle vivait dans un couple heureux et parce qu'elle était une femme très sensible que la tristesse de son enfant touchait profondément, elle est devenue capable de se forger de lui une image plus réaliste. Néanmoins une telle évolution n'arrive pas toujours.

\section{Violence et abus}

Lorsqu'on évoque la problématique sexuelle en lien avec l'enfance, ce sont les abus sexuels qui automatiquement effleurent l'esprit de nos interlocuteurs. La Maison buissonnière a d'ailleurs gagné un prix discerné par un organisme qui fait la promotion de la protection des enfants contre de telles pratiques perverses, malgré que les donateurs n'eussent pas vraiment compris comment le travail centré sur l'enfant, c'est-à-dire sur l'écoute de ses questions, pouvait prévenir des dérives futures lorsque les enfants deviendraient grands. Avec deux exemples tirés de ma pratique en bureau privé, voici un aperçu de ce que le travail enfants-parents peut aider à éviter à plus long terme.

La maman de V. me téléphone catastrophée. Sa fille de 10 ans, qui a toujours été joyeuse et respirait le bonheur de vivre, est devenue triste, pleure souvent, se trouve laide et estime presque que la vie ne vaut pas la peine d'être vécue. Elle semble avoir vécu des grosses déceptions dans ses relations avec ses amies à l'école. La maman, qui dans son enfance a été le souffre-douleur de sa classe, ne se sent pas en mesure de l'aider. Le papa, qui dit adorer sa fille et ne rien comprendre de ce qui lui arrive, souhaite aussi que je puisse la rencontrer : ses conseils qui se résument à des injonctions de ne pas s'en faire et des bons mots restent sans effet. V. veut venir me voir et ne veut surtout pas que les parents l'accompagnent.

Je vois arriver une jolie fillette énergique et coquette, qui ne me donne pas l'impression d'un être profondément déprimé. Son premier dessin — une marguerite gracieusement dressée et pleinement épanouie — renforce mon impression. V. me raconte alors la situation qui règne sur la cour de son école. Les élèves y sont répartis en trois groupes, territorialement répartis. Le premier, celui qui compte, est constitué d'une grappe de filles agglutinée autour de la Lolita de la place. Cette fillette qui porte des cheveux oranges et un faux tatouage en haut de fesses, est sensée connaître la vie et savoir les choses sur la sexualité. Elle trouve risible et infantile la bonne humeur de V. et ne l'accepte pas pleinement dans son groupe. Son supposé savoir sur la sexualité repose sur sa situation d'enfant d'un couple séparé qui a déjà connu un certain nombre de beaux-pères. Un deuxième 
groupe de filles est en passe de s'organiser en contre-pouvoir autour d'une autre fillette qui, à 11 ans est déjà menstruée. Ce groupe est moins structuré, mais reste un peu ambivalent quant à l'acceptation de V. en son sein. Par contre, sa meilleure amie vient de troquer son amitié contre l'honneur d'être reconnue par la fillette menstruée. Le reste de la cour est constitué de garçons qui jouent à la balle et par les filles que cette activité stupide et masculine intéresse. Ce dernier groupe est nommé «nowhere». Cela me prend quelques rencontres pour pouvoir tracer dans mon esprit une image claire de la situation et de comprendre pourquoi la maman et le papa de $\mathrm{V}$. ont de la difficulté de venir en aide à leur fille. Trois ou quatre séances suffisent par la suite pour lui faire reconnaître certains de ses sentiments et voir avec elle comment elle aurait pu se sentir, si à la place de son père une succession d'hommes se retrouvait dans le lit de sa mère. Un brin d'éducation sexuelle la libère de l'angoissante perspective d'être forcée à une sexualité précoce.

Il est déconcertant de constater quotidiennement à quel point notre société tient un discours double sur la sexualité. D'une part, celle-ci doit être libre. Elle est montrée (à la télévision, sur les panneaux publicitaires), parlée (dans les revues), fait partie des biens consommables. Les expériences sexuelles deviennent un must pour des adolescentes de plus en plus jeunes (12,13 ans) qui veulent rester in. Les cours des écoles primaires dans les quartiers cossus autant que pauvres se marquent de divisions invisibles pour les enseignants. Celles (puisqu'il s'agit de filles) qui comptent (sont populaires, parce que censées posséder un savoir sur la sexualité et censées la vivre) mettent au ban les autres, aspirantes non élues pour partager des secrets sexuels, lesquelles dépriment. Dès 13, 14 ans, les filles se lancent à l'assaut d'expériences sexuelles pour lesquelles elles ne sont pas préparées, mais qu'elles pensent nécessaires pour garder l'estime de soi et celle des autres. D'autre part, les adultes ne se sentent pas du tout libres de discuter de questions sexuelles avec leurs enfants, même quand eux-mêmes sont heureux dans leur couple. En fait, malgré tous les signes que les enfants leur adressent, ils s'obstinent à ne pas voir.

V. a rapidement retrouvé sa bonne humeur, a repris ses occupations parascolaires et a récupéré sa «meilleure amie». Elle se trompait néanmoins en attribuant une innocence infantile généralisée et méprisable au groupe de garçons indifférenciés du «nowhere».

Les parents du petit S. (7 ans et demi) viennent me voir parce que la mère n'est pas d'accord avec la prescription de rhitalin qu'il vient de recevoir. L'enfant est décrit comme intenable à l'école. Le père pense qu'il est effectivement un peu agité, mais 
trouve que c'est normal pour un petit garçon. Ils ont aussi un fils aîné, de deux ans plus âgé que S., qui ne présenterait pas de problème. Très rapidement, il apparaît que le couple parental ne va pas bien du tout. La mère de $\mathrm{S}$. veut quitter la famille. Elle a beaucoup de reproches à adresser au père - entre autres sa passivité, son manque d'ambition et l'insatisfaction sexuelle qu'elle éprouve. Le père semble un peu dépassé par les événements, mais sans plus. Les rencontres avec $\mathrm{S}$. en présence de sa mère qu'il ne veut pas quitter, avec les parents seuls, avec toute la famille sont vraiment chaotiques et je me demande où tout cela va nous mener jusqu'au moment où $\mathrm{S}$. se dessine avec une énorme main rouge. Il accepte alors de poursuivre seul avec moi.

Dans les séances qui suivent, j'apprends qu'à la maison, les amis de son frère jouent avec lui au docteur en lui mettant des crayons dans les fesses, et qu'à l'école (une petite école privée) les amis de classe de son frère le poursuivent pendant les recréations pour lui baisser ses pantalons et le masturber. Bien qu'il ne déteste pas ces jeux et se masturbe lui-même, il trouve qu'à la fin cela fait mal et préfère passer ses récréations ainsi que la période où il attend sa mère qui vient le chercher à l'école, sur une chaise devant le bureau de la directrice. Quand je convoque une rencontre familiale pour discuter de tout cela, le père ne veut pas croire ses oreilles, même s'il se rappelle vaguement que les garçons étaient venus lui demander des crayons et qu'il n'avait pas très bien compris pourquoi ils trouvaient que les leurs ne sentaient pas bon. Il sort de sa léthargie, met de l'ordre dans la vie sexuelle débridée de ses enfants autant à la maison qu'à l'école, jette le rhitalin dans les toilettes et demande une thérapie pour lui-même. C'est aussi lui-même qui répondra à toutes les questions que ses garçons vont par la suite lui poser.

Rassurée par le fait qu'il se trouve alors «entre les bonnes mains », la mère quitte le domicile familial pour poursuivre un amant qu'elle a connu brièvement à l'étranger. À cause de sa préoccupation de ne pas répéter une histoire familiale fort mouvementée sur le plan affectif et sexuel, elle n'avait pas quitté la famille où, dit-elle, il fallait qu'elle s'occupe de tout. Maintenant elle nous quitte, son mari et moi-même. Je ne la reverrai d'ailleurs plus jamais, mais elle reviendra quand même assez rapidement à Montréal et établira un rythme de visites pour les enfants. Le père me quittera finalement à son tour après avoir fondé une famille «reconstituée». 
La violence dans des relations entre enfants reflète celle qu'ils ressentent non seulement à cause de difficultés parentales, mais surtout à cause du silence qui les accompagne. Vers l'âge de 13, 14 ans lorsque non seulement les questions fondamentales des enfants restent toujours sans réponse, mais que l'on continue de les laisser à eux-mêmes, abus et violences graves peuvent faire leur apparition.

\section{La contestation des contraintes sociales : entre liberté et délinquance?}

Autant que Françoise Dolto jugeait nécessaire de «parler vrai» aux enfants, autant elle faisait la démonstration de la nécessité de les faire accéder à la série de ce qu'elle appelait «les castrations ». Recevoir les «castrations » permet à l'enfant de parcourir sans encombre les étapes de son développement affectif et devenir un citoyen civilisé, c'est-à-dire de renoncer définitivement aux modes relationnels périmés alors qu'il est en mesure d'en conquérir d'autres, plus adéquats pour l'étape de son développement selon l'âge. Appliquer ces «castrations » de façon souple et sans violence fait partie de la fonction éducative des parents. Ceci n'est possible que si les parents eux-mêmes ont abandonné au cours de leur développement des modes de fonctionnement basés sur la toute puissance infantile.

Or la société post-moderne ne favorise nullement de tels renoncements. Elle fait la promotion du développement individuel au détriment de la cohésion sociale et privilégie la satisfaction du désir au détriment du processus de deuil. La révolution contraceptive, qui a permis de séparer la sexualité de la procréation, a ouvert la voie à la constitution d'un leurre : celui de la possibilité réelle de déliaison de la maternité et de la sexualité avec, comme conséquence, le démantèlement fantasmatique de la triade initiale : mère, père, enfant.

D'une configuration où la société présentait à tout nouveau-né un champ restreint de possibles pour son devenir en le subordonnant à un ordre social basé sur l'ordonnancement des sexes, des âges et des classes, on est passé à une autre où, dans un effort d'égalitarisme sans précédent, notre société actuelle tente d'effacer l'un comme l'autre les axes de cet ordonnancement. L'utopie actuelle commande à chacun de suivre son désir. Du côté éducation, la contestation des contraintes sociales s'est soldée par l'abandon de l'enseignement de ce qu'on appelait banalement dans le temps les règles sociales de bienséance. Ces règles, qui servent de pacificateurs du lien social, marquent habituellement la place de chacun dans l'ordre social selon l'ordre des générations et donc selon l'âge, le sexe, le statut hiérarchique et le contexte de rencontre. Pour les appliquer, il faut d'abord les intérioriser et les partager soi-même. Pourtant de plus en plus d'adultes restent en panne de référence à une norme partagée par la société dans laquelle ils vivent lorsqu'ils se trouvent confrontés à des situations nouvelles ou qui leur sont inhabituelles, c'est-à-dire lorsqu'ils sortent de l'univers domestique ${ }^{9}$, lourds et maladroits comme des touristes dans une culture inconnue. Pour pouvoir fonctionner dans une société, l'enfant doit non seulement apprendre et intérioriser les lois et les règles domestiques, particulières d'une famille à l'autre, mais aussi les lois et règles générales de co-existence dans la société qui est la sienne. Il lui faut 
apprendre à différencier la sphère domestique et les divers niveaux de règles de coexistence sociale. Il doit apprendre des codes qui lui permettent de déchiffrer dans quel genre de relation sociale il se trouve.

La promotion médiatique du bonheur a eu comme effet de déprécier les voies de la sublimation, qui exigent la suspension et la mise en attente de la satisfaction immédiate. De plus en plus de parents, qui ne sont pas eux-mêmes délinquants ou ignorant du savoir-vivre, craignent de «traumatiser» l'enfant ou de perdre son amour s'il lui imposent la suspension de la satisfaction du désir, ou simplement, lui imposent des limites. Ils n'arrivent alors que difficilement de lui dire non ou ne peuvent le dire que lorsqu'ils sont au bout et deviennent agressifs.

Une des tactiques que les adultes adoptent lorsqu'ils sont confrontés à la nécessité de socialiser l'enfant est l'illusion qu'il «ne comprend pas» les injonctions. Le «monde sans limites» qui devient celui de l'enfant se peuple alors de dangers internes; son chaos émotionnel se manifeste par des comportements de retrait ou de violence. Sans limites, livré à l'arbitraire de la mère ou de ceux qui l'élèvent, l'enfant n'est certainement pas préparé pour affronter la vie sociale et ne peut pas aller à la rencontre des autres — il ne peut éventuellement que se heurter à eux ou apprendre à les utiliser pour sa satisfaction comme il peut être lui-même instrumentalisé pour satisfaire des tendances inavouées de ceux qui s'occupent de lui.

$\mathrm{X}$. fréquente plus au moins assidûment La Maison buissonnière depuis déjà quelques mois, accompagné de sa grand-mère. À presque deux ans, il est très costaud. Il ne prononce encore aucun mot, ne s'intéresse pas aux autres enfants, utilise sa force pour obtenir ce qu'il désire, mais nous n'avons aucun doute qu'il s'agit d'un enfant intelligent. À l'occasion d'une visite, prend place une sorte de confrontation entre sa grand-mère et le personnel d'accueil autour de la question des limites. Alors que $\mathrm{X}$. essaie de traverser la ligne qui sépare le local en deux en tirant un véhicule porteur à grandes roues, ce qui n'est en aucun cas autorisé, sa grand-mère trouve que nous sommes vraiment ridicules de croire qu'il pourrait comprendre l'interdiction. Nous apprenons par la même occasion que chez lui il déplace les meubles et que c'est une vraie terreur lorsqu'il décide, en visite chez elle, de fouiller dans des objets mis à sa hauteur. Comme nous nous étonnons qu'on le laisse faire de telles choses, elle nous répond que, dans les espaces restreints des appartements, il faut bien qu'il se trouve quelque chose à faire! Sa satisfaction, que les exploits du petit garçon provoquent chez elle, est facilement perceptible. Pendant notre discussion avec sa grandmère, $\mathrm{X}$. essaie de traverser la ligne de plusieurs façons: en tirant et en poussant le véhicule, en s'asseyant dessus, en le portant sous le bras, puis finalement, face à notre refus de le 
laisser passer malgré tous ses astuces, il abandonne. Cela n'empêche pas la dame, qui tient à son «il ne comprend pas », d'affirmer qu'il est peut-être moins intelligent que nous ne le pensons. Surprise elle-même par ce jugement négatif qu'elle porte sur son petit-fils, elle change alors de tactique et se met à nous questionner sur nos qualifications. Nous avons pourtant le sentiment qu'au fond, elle a dit vrai sur la représentation de cet enfant qu'elle-même ou la famille tiennent à maintenir. Quelles satisfactions cachées peut-elle en tirer?

À La Maison buissonnière plusieurs règles constituent le cadre de rencontres. Certaines d'entre elles s'adressent aux parents seuls (laisser un paiement, inscrire le prénom de l'enfant), d'autres s'adressent à l'enfant, mais concernent tout le monde puisque autant les parents que les personnes d'accueil doivent veiller à leur respect et les respecter elles-mêmes. Nous constatons que, parfois, il nous est plus difficile de faire comprendre la nécessité de respecter notre cadre aux parents qu'aux enfants de deux ans - et pourtant on prétend que c'est un âge de contestation, l'âge du «non»...

Le petit Y. a fréquenté La Maison buissonnière pendant plusieurs années. Nous avons fait sa connaissance alors qu'il avait quelques mois et il nous a quittés au moment de l'entrée à la prématernelle. Pendant au moins une année et demie, sa maman a testé, en les dépassant plus au moins subtilement, toutes nos limites. Par exemple, elle n'enlevait pas ses chaussures d'extérieur, partait souvent la dernière en retardant la fermeture du local ou permettait que son enfant se promène partout avec de la nourriture dans les mains, ce qui faisait ressembler la pièce à une mangeoire d'oiseaux. Il était très rare que la maman de Y. nous dise bonjour en arrivant, alors qu'elle s'installait confortablement au milieu de la pièce. Judoka dans ses moments libres, elle a mis K.O. tous les hommes de l'équipe sans user d'autres prises que ses attitudes et, parfois, ses remarques assassines. Il nous a fallu montrer une certaine fermeté, mais en même temps ne pas faire preuve de rigidité dans le maintien des règles pour que, progressivement, elle les fasse siennes. Exactement de la même façon que les petits.

La maman de Y. était en admiration sans bornes devant son fils, qu'elle contrôlait néanmoins sévèrement s'il enfreignait ses règles personnelles à elle. Il leur arrivait de se chamailler, mais elle finissait par couper court et avec une certaine violence retenue à ces confrontations. C'était elle le boss. L'enfant avait l'habitude d'être obéissant lorsque sous le regard de sa mère, 
Moi, toi et... ou est passé le roi ? Les voies de la socialisation précoce

mais essayait toutes les sortes de mauvais coups quand elle ne le surveillait pas. Il avait développé une agilité surprenante et, pour nous, étourdissante, ainsi qu'une résistance aux chocs. À la grande satisfaction de sa mère, il ne pleurait presque jamais en tombant. Pendant très longtemps, il nous a été impossible de nous adresser à lui directement. Mon habitude de fréquenter des délinquants et une réelle sympathie pour elle et son enfant semblent avoir été les premiers éléments qui ont permis de briser un peu la glace. C'est seulement après avoir développé une sorte de relation identificatoire avec certaines d'entre nous (au point de nous renvoyer notre image en miroir lorsqu'elle s'est mise à intervenir auprès des autres enfants présents), qu'il nous a été possible, à notre tour, de nous adresser à Y. Mais encore là, la mère était très vigilante et susceptible; elle craignait qu'on la critique ou qu'on trouve l'enfant fautif lors de heurts avec les autres. En grandissant, l'enfant s'est calmé et est devenu industrieux. Quelques mois avant la fin de leur fréquentation du centre, donc dans la perspective d'une séparation imminente, la maman a pu nous dire l'importance que disposer de ce lieu a eu pour son enfant et pour elle-même.

«Les comportements d'incivilité, et parfois de violence, sont bien l'expression d'une tentative d'inviduation qui se fait en opposition aux adultes, comme le passage d'adolescent l'impose, mais sur un modèle de différentiation agressive hérité des processus infantiles » écrit Serge Lesourd. (2006, p. 27). Il semble que La Maison buissonnière ait servi à cette mère pour effectuer ce passage, ce qui nous a ensuite permis d'introduire dans l'univers de l'enfant les lois (ou les règles) qui n'étaient plus l'œuvre exclusive de l'omnipotence maternelle.

\section{Le «parler vrai » et les pièges du langage}

Seulement 2 à $3 \%$ de l'inscription génétique sépare l'être humain du singe le plus proche. Ce petit pourcentage contient néanmoins la capacité de parole, qui différencie à jamais le bébé humain du bébé-singe (Diamond, 1992).

La parole plonge d'emblée le bébé humain dans l'univers symbolique et structure aussi bien sa pensée consciente qu'elle organise son inconscient. Être de culture, son cerveau inscrit dans la matérialité de ses structures corticales les expériences de ses premières années. Son cerveau fixe ainsi dans sa matérialité des liens entre ses expériences affectives et des mots (dans leurs aspects sensoriels et abstraits) qui s'agencent en langage.

Ces quelques dernières années, nous assistons à des progrès des connaissances issues des expériences sur la compréhension du langage parlé par les nourrissons (De Boysson-Bardies, 1996). Ce que les mères pressentaient et que Françoise Dolto affirmait depuis longtemps s'avère finalement confirmé de façon jugée 
suffisamment objective - le bébé humain a une capacité très précoce de comprendre ce qu'on lui dit. Cette capacité précède de beaucoup la maturation de son système phonatoire, qui lui permet ensuite de parler. Il a aussi une capacité (probablement innée) de créer des catégories et des concepts.

La communication langagière a une fonction tierce; elle permet de sortir du corps à corps avec la mère. Elle est le marqueur de l'appartenance de l'enfant au groupe social qui parle sa langue. Adultes comme enfants, nous ne pouvons néanmoins généralement pas penser ce qui ne fait aucunement partie de notre expérience, ce qui est radicalement inconnu. La pensée de l'enfant est étroitement liée à ses expériences corporelles qui sont l'univers à partir duquel il interprète ce qu'il perçoit du monde qui l'entoure. L'état émotionnel de ses proches et les mots qu'ils lui adressent lui servent de guide.

D'un côté, lorsque les choses ne sont pas nommées, leur existence est incertaine, évanescente. D'un autre côté, la langue est elle-même porteuse de malentendus. Non seulement toute langue génère des phrases ambiguës (qui peuvent recevoir plusieurs interprétations) mais encore elle utilise des formes indirectes d'expression, métaphore, métonymie et d'autres figures de rhétorique. Ne pas se contenter de comprendre littéralement ce qui est dit est une compétence qui doit s'acquérir; il faut du temps à l'enfant pour y arriver. Il ne comprend ni ne manie spontanément l'ironie, le sarcasme, le double sens ou d'autres jeux de langage indirects. Il est donc souvent difficile aux adultes de se rendre compte des malentendus qu'ils provoquent simplement par leur façon de parler.

La communication langagière passe aussi par le filtre de l'expérience subjective. Toute communication humaine est teintée de projection et toute possibilité de communication repose sur les processus de l'identification. C'est pourquoi il est si difficile d'appliquer, même à ceux qui le veulent absolument, l'impératif de Dolto de «parler vrai» à l'enfant. Parler vrai suppose non seulement une liberté intérieure de l'adulte mais sa capacité d'énoncer la vérité qui est celle de l'enfant à un moment donné de son développement et dans une situation précise.

Arriver au plus proche du «parler vrai» aux enfants, rendre le parent apte à une telle prouesse, tel est l'objectif de travail avec ceux qui traversent le seuil de nos deux points d'accueil. Le processus de socialisation précoce commence avec l'écoute et la parole et ne repose pas sur les savoir faire inculqués. Vouloir remédier aux difficultés entre la mère et son enfant en apprenant à la mère des gestes et des comportements adéquats, ce vers quoi semblent mener les applications récentes de la théorie de l'attachement dans le réseau de santé au Québec est un non-sens. Reste à espérer que la relation transférentielle que les mères vont nécessairement développer avec les personnes chargées de ces tâches leur permettra d'en tirer un certain profit.

\section{La socialisation précoce}

C'est en étudiant les névroses que Sigmund Freud s'est tourné vers une pièce de Sophocle pour nommer «complexe d'EEdipe» le processus qui ouvre la voie au 
devenir social du petit garçon; la sexualité et la culpabilité sont alors au cœur de sa réflexion. Plus d'un demi-siècle plus tard, Jacques Lacan a abordé la question du père à partir de la psychose. La terreur et l'angoisse président à la «forclusion du nom du père» qui laisse l'enfant démuni et pris dans la relation duelle à la mère. La psychose a toujours existé et continue d'exister, quelle que soit la réalité manifeste de la constellation familiale; par contre, nous rencontrons aujourd'hui de moins en moins de névroses franches. Nous devons réfléchir à partir d'états incertains dits «états limites», personnalités narcissiques, états dépressifs chroniques. Chez les enfants, diverses symptomatologies liées à l'angoisse se manifestent de façon massive. On tente de les museler en diagnostiquant à plein régime «hyperactivité», TOC, difficultés d'apprentissage. Cette labilité des symptômes individuels répond aujourd' hui à l'absence de normes claires régissant les rapports entre individus dans la société. Liberté personnelle conjuguée à accommodements raisonnables ne permettent guère d'établir de référents clairs.

Depuis Mélanie Klein, la psychanalyse nous enseigne que le développement du surmoi névrotique atténue la férocité du surmoi précoce. Si les lois sociales, qui se matérialisent dans les règles de vie, constituent un cadre suffisamment solide à l'existence de l'enfant, elles lui épargnent les angoisses liées au surmoi précoce et lui permettent d'économiser son énergie, qu'il peut alors appliquer ailleurs: à rêver, à apprendre, à jouer.

Lorsque le cadre de co-existence sociale devient flou, la liberté individuelle semble plus grande, mais la porte ouvre sur la possibilité d'angoisse et de la violence. Cette violence n'est pas nécessairement sanglante. Elle peut prendre des formes subtiles, se cacher derrière des objectifs le plus nobles. C'est ce que nous observons tous les jours dans notre travail avec les enfants et les parents. C'est pourquoi cette pratique centrée sur l'écoute des tout petits nous amène à réévaluer la pertinence des arguments avancés dans la discussion autour des enjeux sociétaux actuels. Cette lorgnette peu commune organise des pans entiers du débat souvent chaotique.

\begin{abstract}
Alors?
Les avancées technologiques et scientifiques ouvrent des voies nouvelles à l'organisation de la société. Tout n'est pas toutefois nécessairement entièrement nouveau sous le soleil. Les familles «reconstituées » ont existé pendant des siècles à cause de la mortalité précoce; les familles où le nombre des enfants était très réduit ont été tout aussi nombreuses pour la même raison; la conditions des femmes a fluctué avec les époques et les pays, tout comme des mesures d'éducations.

À l'époque où avait cours la complainte du père absent, alors que l'ambivalence avait commencé à se faire sentir entre l'énoncé de cette doléance et le désir d'affirmer qu'à l'enfant la mère seule suffisait, la question suivante m'a été posée dans un colloque: «Pourquoi ne pourrait-il pas suffire que la relation entre l'enfant et sa mère soit triangulée par l'intérêt que cette dernière porterait à son travail ou
\end{abstract}


aux autres sphères de la vie culturelle?». Un peu prise de court, j'avais alors répondu qu'on ne pouvait pas faire un enfant avec son travail et... je me suis fait huer par l'assistance. Cette question tentait d'introduire l'équivalence entre l'investissement de soi dans un engagement culturel et la relation amoureuse basée sur le désir sexuel. La sublimation est certes une expression des plus élevées de notre humanité; il n'en reste pas moins que dans une telle configuration fantasmatique, qui élimine le partenaire de l'union qui a permis la naissance de l'enfant ou qui efface la sexualité adulte de la mère, «les enjeux sont de rivalité et de pouvoir entre la mère et l'enfant et ensuite entre les instances éducatives et l'enfant», ainsi que le souligne justement Lesourd (2006).

Ma pratique d'accueil dans une perspective de socialisation précoce a eu une influence décisive sur ma pratique clinique autant avec les enfants qu'avec les adultes. C'est le cas pour tous ceux qui travaillent avec les nourrissons, des petits enfants et leurs proches à La Maison buissonnière. Après 15 ans d'expérience et plus de 30000 visites d'enfants de moins de 4 ans, je suis persuadée de son efficience préventive pour divers malaises de l'enfant, voire pour des problèmes majeurs qui peuvent rapidement atteindre son fonctionnement affectif, intellectuel et social. La prévention passe par ce double accueil — de l'enfant et de ses proches — dans un espace aménagé de façon à permettre à la mère, au père et à l'enfant de trouver leurs repères.

Ce qui est vraiment nouveau dans cet accueil est le fait d'ériger l'enfant en interlocuteur principal. Il y est accueilli avec ses proches comme sujet de parole. La famille, voire la société, sont alors interrogées autant que possible, depuis sa place à lui. Les débats qui concernent les valeurs sociétales, la délinquance, l'éducation et la psychopathologie trouvent donc leur point de convergence dans ce concept de socialisation précoce que la Maison Verte — et La Maison buissonnière à sa suite — ont mis au centre de leurs réflexions. Et alors voilà. «Moi, toi et le roi, ça fait trois » disait une ancienne comptine. Mon cheminement aboutit à la constatation que, sans une prise en compte du cadre sociétal, du père et du lien entre la sexualité et la maternité, il est difficile de penser à un travail de prévention auprès de jeunes familles. Non sans humour, les enfants d'antan ne chantaient-ils pas aussi :

«Savez-vous planter les choux, à la mode, à la mode, Savez-vous planter les choux, à la mode de chez nous?»

irène krymko-bleton département de psychologie université du québec à montréal cp 8888, succ. centre-ville montréal qc h3c 3p8 
Moi, toi et... ou est passé le roi ? Les voies de la socialisation précoce

\section{Notes}

1. Pour information voir le site www.maisonbuissonniere.org qui présente son fonctionnement et ses assises théoriques.

2. La psychanalyse - hors la cure — est la réponse qu'a proposée Françoise Dolto pour répondre aux difficultés rencontrées par les jeunes familles. Dans une perspective de prévention primaire, elle souhaitait ainsi «faire communiquer parents et enfant, bien avant qu'il n'y ait un symptôme fixé» (Dolto, 1987). Ses concepts d'image inconsciente du corps et de castrations symboligènes se trouvent aux fondements de cette pratique. Pour en savoir plus, Dolto 1981, 1985, 1987.

3. Les principes de fonctionnement de la Maison buissonnière ainsi que la pratique clinique qui s'y développe ont été décrits à plusieurs reprises, notamment dans le vol. 10 de Filigrane: Krymko-Bleton I. (2001). Voir aussi : Krymko-Bleton I. (2001) et Malandrin, M-H., Krymko-Bleton I., El Khouri M-F., Perron S. (1997).

4. Pour les relations réciproques entre psychanalyse et théorie de l'attachement, voir Krymko-Bleton I. (2007).

5. François Cardozo.

6. Ainsi, elle enchâsse familles et individus dans le tissus social. Si l'on suit l'évolution des sociétés sous cet angle, on s'aperçoit aisément que plus une société est hiérarchisée, plus la famille reproduit en son sein cette hiérarchisation et moins les institutions administratives et législatives sociétales interviennent dans la façon dont elle gère ses membres. Plus une société est égalitaire, plus les relations intrafamiliales sont soumises à l'arbitrage extérieur. Dans la société occidentale contemporaine, cet arbitrage est fréquemment fondé sur des savoirs «scientifiques » du moment. Ainsi nombre d'experts se substituent de façon plus au moins directe tant à l'autorité parentale qu'à l'autorité législative. Habituellement, ces experts ne constituent néanmoins pas un tiers suffisamment structurant pour infléchir l'évolution psychique d'un enfant. Ils se cachent d'ailleurs derrière un savoir «objectif» du moment qui les rallie aux idéologies en cours et qui les protège de remises en question personnelles.

7. Selon Statistiques Canada, un quart des enfants de 7 ans vit actuellement une situation de séparation de ses parents. La moitié de ces séparations surviennent pendant les deux premières années de vie de l'enfant.

8. Sociologue et chercheur au Centre d'études appliquées sur la famille à l'école de service social de l'Université McGill

\section{Bibliographie}

Bleton I., 1989, L'éternel absent: les pères à l'ancienne et les nouveaux pères au Québec, Dialogues — recherches cliniques et sociologiques sur le couple et la famille. 104, $\mathrm{n}^{\circ}$ thématique «Cherche père désespérément: nouvelles conceptions, nouveaux pères?». Paris, éd. A.F.C.C.C,. p. 85-93.

De Boysson-Bardies B., 1996, Comment la parole vient aux enfants, Paris, Éditions Odile Jacob

Diamond J., 1992, The Third Chimpanzee. The Evolution and Future of the Human Animal, New York, Harper Perennial.

Dulac G. 2000, La fragilité de la paternité dans la société québécoise: les paradoxes du père nécessaire et du père abject, Défi jeunesse, 6, 3 .

Dolto F., 1987, Tout est langage, Paris, Carrère.

Dolto F., 1985, Nous irons à la Maison Verte, in La cause des enfants, Paris, Robert Lafont.

Dolto F. 1981, La Maison Verte, un lieu de vie, in La difficulté de vivre. Le psychanalyste et la prévention des névroses, Paris, InterÉditions.

Gagnon J-P., 2006, Consultation portant sur la paternité du Conseil de la famille et de l'enfance.

Krymko-Bleton I., 1990, Le père écarté : la complainte du père absent dans la famille et la société québécoise, in : $L a$ Famille: l'individu plus un. Approche psychanalytique et approche systémique (sous la dir. de C. Brodeur, R. Pelsser \& G. Tarrab), éd. Vermette inc., Boucherville, p. 227-255. 
Filigrane, automne 2007

Krymko-Bleton, I., 1985, La malprise des pères, Santé mentale au Québec, vol. X (1). p. 15-19.

Krymko-Bleto,n I., 1990, Le père confus, PRISME, 1,1, p. 46- 53

Krymko-Bleton, I., 2001, La Maison buissonnière: conversations polyphoniques avec des tout petits, Montréal Filigrane, 10.

Krymko-Bleton, I., 2001, Quand les tout petits quittent la maison : l'expérience de la Maison buissonnière, Montréal PRISME, 35

Lacharité, C., 2004, La paternité : enjeux sociaux pour les psychologues, Psychologie Québec, 29.

Lesourd, S., 2006, Comment taire le sujet? Des discours aux parlottes libérales, Ramonville, Érès, coll. Humus

Malandrin, M-H., Krymko-Bleton, I., El Khouri, M-F., Perron, S., 1997, Entre l'espace psychique et l'espace social : le dispositif de travail avec les tout-petits et leurs familles, in Transports de psychanalyse, coll. sous la direction de Krymko-Bleton I, Montréal, L'Harmattan.

Martinat, S., 1990, Actes du père et l'inconscient religieux au Québec, in collectif La famille: l'individu plus un. Approche psychanalytique et approche systémique (sous la dir. de C. Brodeur, R. Pelsser \& G. Tarrab), éd. Vermette inc., Boucherville, p. 257-283

Martinat, S., 1987, Un baptême de père, in collectif Cour-Atout. Un amour de père, Montréal. Éditions Saint-Martin, p. $223-228$

Martinat, S., 1985, Des influences du religieux et de ses thèmes dans la formation du concept de paternité dans la famille québécoise contemporaine, thèse de doctorat, UQAM.

Winnicott, D.W., 1969, L'esprit et ses rapports avec psyché-soma. [1949], L'agressivité et ses rapports avec le développement affectif, [1950-1955], La première année de la vie [1958] in De la pédiatrie à la psychanalyse, Paris, Payot. 